\title{
MULTIPLIERS FOR THE SPACE OF ALMOST- CONVERGENT FUNCTIONS ON A SEMIGROUP
}

\author{
CHING CHOU AND J. PETER DURAN
}

\begin{abstract}
Let $S$ be a countably infinite left amenable cancellative semigroup, $F L(S)$ the space of left almost-convergent functions on $S$. The purpose of this paper is to show that the following two statements concerning a bounded real function $f$ on $S$ are equivalent: (i) $f \cdot F L(S) \subset F L(S)$; (ii) there is a constant $\alpha$ such that for each $\varepsilon>0$ there exists a set $A \subset S$ satisfying (a) $\varphi\left(X_{A}\right)=0$ for each left invariant mean $\varphi$ on $S$ and (b) $|f(x)-\alpha|<\varepsilon$ if $x \in S \backslash A$.
\end{abstract}

1. Let $S$ be a semigroup, $m(S)$ the space of bounded real functions on $S$ with the sup norm. $\varphi \in m(S)^{*}$ is called a left invariant mean on $S$ if $\|\varphi\|=1, \varphi \geqq 0$ and $\varphi\left(l_{s} f\right)=\varphi(f)$ for $s \in S$ and $f \in m(S)$, where $l_{s} f \in$ $m(S)$ is defined by $\left(l_{s} f\right)(t)=f(s t), t \in S$. The set of left invariant means on $S$ is denoted by $M L(S)$. If $M L(S)$ is nonempty, then $S$ is said to be left amenable [2]. A bounded real function $f$ on a left amenable semigroup is called left almost-convergent if $\varphi(f)$ equals a fixed constant $d(f)$ as $\varphi$ runs through $M L(S)$ [2]. The set of all left almost-convergent functions, denoted by $F L(S)$, is a vector subspace of $m(S)$ and it contains constant functions. But, in general, it is not closed under multiplication. The purpose of this paper is to study this aspect of $F L(S)$ and our main result is the following.

THEOREM. Let $S$ be a countable left-cancellative left amenable semigroup without finite left ideals. Then the following two statements concerning a function $f \in m(S)$ are equivalent:

(i) $f$ is a multiplier of $F L(S)$, i.e., $f \cdot F L(S) \subset F L(S)$;

(ii) $f$ is $S$-convergent to a constant $\alpha$, i.e., for a given $\varepsilon>0$ there exists a set $A \subset S$ such that

(a) $\varphi\left(\chi_{A}\right)=0$ for each $\varphi \in M L(S)$, and

(b) $|f(x)-\alpha|<\varepsilon$ if $x \in S \backslash A$.

2. From now on $S$ will always denote a left-cancellative left amenable semigroup without finite left ideals.

Presented to the Society, January 17, 1972; received by the editors May 31, 1972. AMS (MOS) subject classifications (1970). Primary 43A07; Secondary 46N05.

Key words and phrases. Amenable semigroups, almost-convergence, multipliers, invariant means, weak Cauchy sequences.

(c) American Mathematical Society 1973 
Remarks. (1) A set $A \subset S$ is said to be left almost-convergent if its characteristic function $\chi_{A}$ is left almost-convergent. In this case we denote $d\left(\chi_{A}\right)$ by $d(A)$. Roughly speaking, a set $A \subset S$ is left almost-convergent if it is evenly distributed in $S$ with respect to the semigroup structure and $d(A)$ indicates the density of $A \subset S$. In particular, $d(A)=0$ means that $A$ is sparsely distributed in $S$. (The set $A$ in the statement (ii) of the above Theorem is such a set.) For example, when $S=N$, the additive semigroup of positive integers, a set $A \subset N$ is left almost-convergent if and only if

$$
\lim _{n}(1 / n) \operatorname{Card}(\{k, k+1, \cdots, k+n-1\} \cap A)=d(A)
$$

exists uniformly in $k$ [7].

(2) Since $S$ contains no finite left ideals, $d(B)=0$ for each finite subset $B \subset S$ (cf. [5]). Therefore if $f$ converges to $\alpha$ at infinity, i.e., if given $\varepsilon>0$ there exists a finite set $B \subset S$ such that $|f(x)-\alpha|<\varepsilon$ whenever $x \notin B$, then $f S$-converges to $\alpha$. On the other hand, the space of $S$-convergent functions is much smaller than $F L(S)$. Indeed, $F L(S)$ separates points of $\beta S$ [6], the Stone-Cech compactification of the discrete set $S$, while it is easy to see that $f \in m(S)$ is $S$-convergent if and only if $f$ is a constant on the set

$$
K(S)=\operatorname{cl}_{\beta S} \cup\{\operatorname{supp} \varphi: \varphi \in M L(S)\},
$$

(cf. [1]). Here we consider a bounded real function on $S$ as a continuous function on $\beta S$ and a mean on $m(S)$ as a probability measure on $\beta S$. In particular, if $\varphi \in M L(S)$, supp $\varphi$ denotes the support of the measure $\varphi$.

(3) As in [2], let $E G$ denote the smallest class of groups which contains all finite groups, all abelian groups and is closed under the following four ways of constructing new groups from given ones: (a) subgroup; (b) factor group; (c) group extension; and (d) direct limits.

Each group in $E G$ is amenable and they constitute all the known amenable groups [2]. If we assume that $S$ is an infinite group in $E G$, then a stronger result is known [1]: $f \in m(S)$ is $S$-convergent if and only if

(a) $f \cdot \chi_{A} \in F L(S)$ for each left almost-convergent set $A$,

(b) $f^{n} \in F L(S), n=1,2, \cdots$;

in particular, if $A \subset S$ and $A \cap B$ is left almost-convergent for each $\chi_{B} \in$ $F L(S)$, then $d(A)=1$ or 0 . It is not clear whether our Theorem yields the same conclusion. The proof of our Theorem is completely different from the proof in [1].

3. Proof of the Theorem. (ii) $\Rightarrow$ (i) is easy, cf. [1].

(i) $\Rightarrow$ (ii). Let $f$ be a multiplier of $F L(S)$. To show that $f$ is $S$-convergent, it suffices to show that $f \equiv d(f)$ on supp $\varphi$. We claim that this follows from the following assertion:

( $\alpha)$ If $g \in F L(S)$ and $\varphi \in M L(S)$, then $\varphi(f g)=\varphi(f) \varphi(g)$. 
Indeed, if $(\alpha)$ holds and if $\varphi \in M L(S)$ then

$$
\varphi\left((f-d(f))^{2}\right)=(\varphi(f)-d(f))^{2}=0 .
$$

Therefore $f \equiv d(f)$ on supp $\varphi$ as we wanted.

Proof OF $(\alpha)$. We shall consider $l^{1}(S)^{*}=m(S)$ and $l^{1}(S)^{* *}=m(S)^{*}$. If $\psi \in l^{1}(S)$ and $h \in m(S)$, then $h(\psi)=\psi(h)=\sum_{t \in S} h(t) \psi(t)$. Since $S$ is left amenable and countable there exists a sequence $\varphi_{n}$ in $l^{1}(S)$ such that $\left\|\varphi_{n}\right\|_{1}=1, \varphi_{n} \geqq 0$, and $\lim _{n}\left(\varphi_{n}(h)-\varphi_{n}\left(l_{x} h\right)\right)=0$ for each $h \in m(S)$ and each $x \in S$ [5, Lemma 5.1]. We shall need the following two well-known facts (cf. $[3, \S 9])$ :

$(\beta)$ If $g \in F L(S)$, then $\lim _{n} \varphi_{n}(g)=d(g)$.

( $\gamma) F L(S)=$ the closed linear span of $\left\{l_{x} h-h: x \in S, h \in m(S)\right\} \cup\left\{\chi_{S}\right\}$.

Let $x \in S$ be fixed. Set $\psi_{n}=\varphi_{n} \cdot f-l_{x}\left(\varphi_{n} \cdot f\right)$, i.e., $\psi_{n}(t)=\varphi_{n}(t) f(t)-$ $\varphi_{n}(x t) f(x t), t \in S$. Then $\psi_{n} \in l^{1}(S)$. We claim that $\psi_{n}$ is a weak Cauchy sequence in $l^{1}(S)$. Indeed, if $h \in m(S)$,

$$
\begin{aligned}
\psi_{n}\left(l_{x} h\right) & =\sum_{t \in S}\left(\varphi_{n}(t) f(t) h(x t)-\varphi_{n}(x t) f(x t) h(x t)\right) \\
& =\sum_{t \in S} \varphi_{n}(t) f(t)(h(x t)-h(t))+\sum_{t \in S \backslash x S} \varphi_{n}(t) f(t) h(t) \\
& =\varphi_{n}\left(f \cdot\left(l_{x} h-h\right)\right)+\varphi_{n}\left(f h \cdot \chi_{S \backslash x S}\right) \equiv a_{n}+b_{n} .
\end{aligned}
$$

Note that $f \cdot\left(l_{x} h-h\right) \in F L(S)$, since $f$ is a multiplier of $F L(S)$ and $l_{x} h-h \in F L(S)$. Therefore by $(\beta) \lim _{n} a_{n}=d\left(f \cdot\left(l_{x} h-h\right)\right)$. Note also that $1 \geqq \varphi\left(\chi_{t S}\right)=\varphi\left(l_{t} \chi_{t S}\right) \geqq \varphi\left(\chi_{S}\right)=1$, i.e., $\varphi\left(\chi_{S \mid t S}\right)=0$ for each $t \in S$. Therefore $\chi_{S \mid t S}$ is left almost-convergent to zero. By $(\beta)$ again, we get

$$
\left|b_{n}\right| \leqq\|f\|_{\infty}\|h\|_{\infty} \varphi_{n}\left(\chi_{S \mid t S}\right) \rightarrow 0 \text { as } n \rightarrow \infty .
$$

Therefore we have obtained:

$(\delta) \lim _{n} \psi_{n}\left(l_{x} h\right)=d\left(f \cdot\left(l_{x} h-h\right)\right), h \in m(S)$.

Since $S$ is left-cancellative, each $k \in m(S)$ is of the form $l_{x} h$ for some $h \in m(S)$. Therefore $\lim _{n} \psi_{n}(h)$ exists for each $h \in m(S)$, i.e., $\psi_{n}$ is a weak Cauchy sequence as we claimed. Since $l^{1}(S)$ is weakly sequentially complete $\left[4\right.$, p. 374], there exists $\psi \in l^{1}(S)$ such that $\psi=\lim _{n} \psi_{n}$ in the weak topology. Certainly, $\psi(t)=\lim _{n} \psi_{n}(t)$ for $t \in S$. On the other hand,

$$
\lim _{n} \varphi_{n}(t)=\lim _{n} \varphi_{n}\left(\chi_{\{t}\right)=0,
$$

since $\chi_{\{t\}} \in F L(S)$ and $d\left(\chi_{\{t\}}\right)=0$ (cf. Remark (1)). Hence

$$
\psi(t)=\lim _{n}\left(\varphi_{n}(t) f(t)-\varphi_{n}(x t) f(x t)\right)=0 .
$$

So, $\psi \equiv 0$. By $(\delta), d\left(f \cdot\left(l_{x} h-h\right)\right)=0=d(f) \cdot d\left(l_{x} h-h\right)$. It is of course true 
that $d\left(f \cdot c \chi_{S}\right)=d(f) \cdot d\left(c \chi_{S}\right)$. Hence $(\alpha)$ follows from $(\gamma)$ and the above observation.

\section{REFERENCES}

1. C. Chou, The multipliers for the space of almost convergent sequences, Illinois $\mathrm{J}$. Math. 16 (1972), 687-694.

2. M. M. Day, Amenable semigroups, Illinois J. Math. 1 (1957), 509-544. MR 19, 1067.

3. - Semigroups and amenability, Proc. Sympos. Semigroups (Wayne State Univ., Detroit, Mich., 1968), Academic Press, New York, 1969, pp. 5-53. MR 42 \#411.

3. N. Dunford and J. T. Schwartz, Linear operators. I: General theory, Pure and Appl. Math., vol. 7, Interscience, New York, 1958. MR 22 \#8302.

5. E. Granirer, On amenable semigroups with a finite-dimensional set of invariant means. I, Illinois J. Math. 7 (1963), 32-48. MR 26 \#1744.

6. S. P. Lloyd, Subalgebras in a subspace of $C(X)$, Illinois J. Math 14 (1970), 259-267. MR 41 \#8995.

7. G. G. Lorentz, A contribution to the theory of divergent sequences, Acta Math. 80 (1948), 167-190. MR 10, 367.

Department of Mathematics, State University of New York at Buffalo, AMHERST, NEW YORK 14226

Department of Mathematics, University of Puerto Rico at Mayaguez, Mayaguez, Puerto Rico 00708 